\title{
Aménager une salle de classe dédiée à la formation des enseignants au $21^{\mathrm{e}}$ siècle Réflexions issues d'un projet interdisciplinaire en contexte universitaire
}

Designing a classroom dedicated to teacher training in the $21^{\text {st }}$ century: Reflections from an interdisciplinary project in a university context

\section{Diseñar un aula dedicada a la formación de docentes en el siglo XXI: Reflexiones de un proyecto interdisciplinario en un contexto universitario}

Jérémie Bisaillon, étudiant au doctorat

Université du Québec à Montréal, Canada

bisaillon.jeremie@uqam.ca

Edith Potvin-Rosselet, étudiante au doctorat

Université du Québec à Montréal, Canada

potvin rosselet.edith@courrier.uqam.ca

Alain Stockless, professeur

Université du Québec à Montréal, Canada

stockless.alain@uqam.ca

Léonie Hottote, étudiante à la maitrise

Université du Québec à Montréal, Canada

hottote.leonie@courrier.uqam.ca

Louise Malé-Mole, étudiante au doctorat

Université du Québec à Montréal, Canada

mole.louise@courrier.uqam.ca 
David Allard-Martin, étudiant à la maitrise

Université du Québec à Montréal, Canada

allard martin.david@courrier.uqam.ca

Carlo Carbone, professeur

Université du Québec à Montréal, Canada

carbone.carlo@uqam.ca

\section{RÉSUMÉ}

Le projet de la Classe du futur vise à repenser les salles de classe à la formation initiale et continue des enseignants. Ce projet s'inscrit dans une volonté d'offrir des espaces d'apprentissage flexibles à l'université tout en préparant les enseignants aux défis du $21^{\mathrm{e}}$ siècle. Par un effort concerté, des étudiants issus des sciences du design et de l'éducation ont proposé une esquisse d'aménagement de locaux existants à l'UQAM. L'esquisse présentée dans cet article permet également de réfléchir sur l'intérêt des projets de recherche interdisciplinaires et les caractéristiques d'une classe d'apprentissage actif en milieu universitaire.

Mots-clés : formation des enseignants, aménagement des classes, apprentissage actif, compétence numérique, interdisciplinarité, hybridation

ABSTRACT

The Classe du futur project aims to rethink classroom design in teacher training. This project is in line with the desire to offer flexible learning environments in university settings while preparing teachers for 21 st-century challenges. In a concerted effort, design and education sciences students proposed a classroom planning design of existing space on the university's campus (UQAM). The result of those efforts is presented in this article. It also offers an occasion to reflect on the value of interdisciplinary research projects and classroom characteristics that enable active learning in university settings.

Keywords: teacher education, classroom design, active learning, digital competencies, interdisciplinarity, blended learning

\section{RESUMEN}

El proyecto Classe du futur tiene como objetivo repensar las aulas en la formación inicial y continua de los docentes. Este proyecto se inscribe en el deseo de proporcionar espacios de aprendizaje flexibles en la universidad mientras se prepara a los profesores para los desafíos del siglo XXI. A través de un esfuerzo concertado, estudiantes de las ciencias del diseño y de la educación propusieron un esbozo de diseño para espacios existentes en el campus de la UQAM. El esbozo presentado en este artículo permite también reflexionar sobre el interés de 
los proyectos de investigación interdisciplinarios y sobre las características de un clase de aprendizaje activo en la universidad.

Palabras claves: formación de docentes, diseño de las aulas, aprendizaje activo, competencia digital, interdisciplinaridad, hibridación

\section{Introduction}

La Classe du futur est un projet de recherche universitaire qui vise à imaginer une classe dédiée à la formation des enseignants permettant la diversification des modalités d'enseignement et des méthodes pédagogiques. Ce projet, réalisé par une équipe d'étudiants issus des sciences du design et de l'éducation, a mené à l'élaboration d'une esquisse d'aménagement. La présentation de cette esquisse représente le cœur du présent article et constitue une première étape vers l'aménagement de la classe dans des locaux existants de la Faculté des sciences de l'éducation à l'Université du Québec à Montréal (UQAM). Cet article décrira d'abord le contexte dans lequel le projet s'inscrit et les enjeux auxquels il répond. Ensuite, le processus de création qui a rendu possible la production de l'esquisse sera explicité. Cette section servira à établir les fondements théoriques du projet, un aspect qui a fait partie de la première phase du processus. Finalement, après une présentation des éléments centraux de l'esquisse d'aménagement, l'article se conclut par une réflexion sur le processus de création et les retombées envisageables du projet pour la pratique des enseignants.

\section{Contexte et enjeux liés au projet}

Dans un contexte où les innovations technologiques s'accélèrent, le quotidien et le monde du travail se voient transformés ( $p$. ex. communication par les médias sociaux, télétravail). Les individus sont alors appelés à recourir à des compétences leur permettant de faire preuve d'adaptabilité et de flexibilité devant les changements (Dede, 2010; Global Partnership for Education, 2020). Ces compétences dites du $21^{\mathrm{e}}$ siècle englobent différentes aptitudes dont la créativité, la communication, la collaboration, la résolution de problème, la pensée critique et la maitrise des technologies numériques (Lewin et McNicol, 2015; Joynes et al., 2019). Le développement de ces compétences en classe demande aux enseignants de créer des situations d'apprentissage permettant l'expérimentation et l'exploitation des technologies numériques (Ertmer et al., 2015; Lewin et McNicol, 2015). Le personnel enseignant est ainsi invité à favoriser une pédagogie active qui englobe différentes méthodes pédagogiques ( $p$. ex. apprentissage par problèmes, apprentissage coopératif) favorisant l'engagement dans la tâche et la réflexion sur le processus d'apprentissage (Bonwell et Eison, 1991; Normand, 2017; Prince, 2004). Ces méthodes ont été largement étudiées et la méta-analyse de Freeman et ses collaborateurs (2014) rapporte que les étudiants réussissent mieux lorsqu'ils sont actifs qu'avec des méthodes transmissives.

Afin de favoriser ces méthodes pédagogiques, la formation du personnel enseignant, l'accès aux ressources et la configuration des espaces d'apprentissage devraient être pris en compte (Lewin et McNicol, 2015). D'une part, la formation revêt un rôle central dans la préparation des enseignants puisque ces derniers ne sont pas nécessairement au fait des méthodes pédagogiques qui permettent d'optimiser l'usage des technologies numériques et de développer les compétences du $21^{\mathrm{e}}$ siècle (Ertmer et al., 2013; Lewin et McNicol, 2015). D'autre part, l'accès aux technologies numériques et les espaces d'apprentissage viennent soutenir ou contraindre certaines méthodes pédagogiques (Cleveland, 2011; Paquelin, 2017). Pour Céci et Coudrin (2014, p.5), le contexte technologique actuel et la nécessité de rendre l'apprenant 
actif amènent à « développer des espaces d'apprentissages permettant le travail de groupes, la mobilité, la collaboration et la coopération [ainsi que] des usages efficaces du numérique [...] ». Ces espaces apparaissent particulièrement pertinents pour la formation du personnel enseignant. En effet, ils peuvent s'avérer des environnements propices pour expérimenter une diversité de méthodes pédagogiques et de technologies numériques. Le projet de la Classe du futur participe ainsi à la réflexion sur les conditions favorisant l'apprentissage en s'interrogeant sur les espaces qui sont dédiés à la formation du personnel enseignant, un contexte qui semble avoir été peu étudié. Le projet permet de placer les principaux utilisateurs de la classe, les étudiants, au centre du processus de création qui sera présenté dans la section suivante.

\section{Phases du projet et processus de création}

La Classe du futur est un projet de cocréation interdisciplinaire qui prend appui sur des assises théoriques. L'ensemble du processus de création a été supervisé par deux professeurs et réalisé par la collaboration étroite entre des étudiants issus des sciences de l'éducation et du design. Le projet s'est déployé en trois phases qui sont décrites dans cette section et résumées à la Figure 1.

\section{Figure 1}

Phases du projet

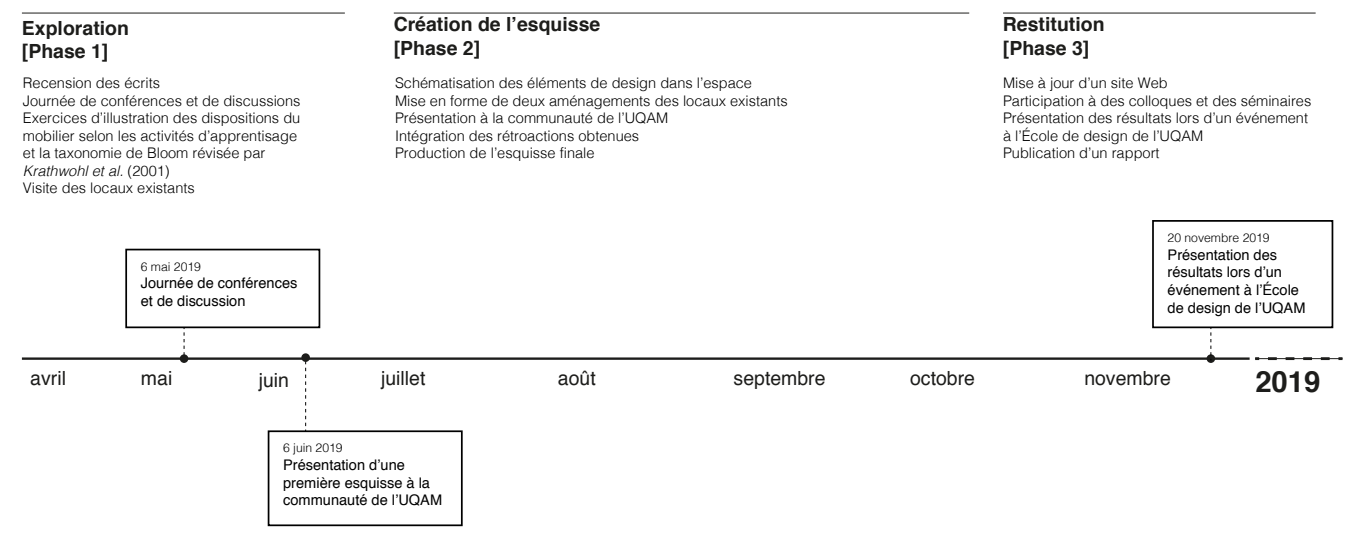

Avant le début de la création de l'esquisse, une recension des écrits sur les classes d'apprentissage actifs à différents ordres d'enseignement a été effectuée. Des rapports et sites Web de projets déjà réalisés au Québec ont également été consultés (Collège LaSalle, 2020; Fournier St-Laurent et al., 2018; McGill, 2020; Université Laval, 2013). II a été possible d'identifier certaines caractéristiques de ces classes. Premièrement, l'espace est souvent divisé en zones à l'aide de cloisons ou par des couleurs qui délimitent différents espaces de travail (Céci et Coudrin, 2014; Barrett et al., 2015; Fournier St-Laurent et al., 2018). Deuxièmement, différentes surfaces d'écriture sont mises à la disposition des apprenants (table, tableau blanc, mur, etc.) (Barrett et al., 2015; Collège LaSalle, 2020; Fournier St-Laurent et al., 2018). Troisièmement, une variété d'outils numériques sont disponibles pour favoriser la présentation des informations, la collaboration et la connectivité (Céci et Coudrin, 2014; Fournier St-Laurent et al., 2018). Quatrièmement, un mobilier (table, chaise, tableau) compact et sur roue permet une reconfiguration rapide de l'espace (Céci et Coudrin, 2014). Finalement, la température et la luminosité sont également identifiées comme des éléments à prendre en compte dans l'aménagement d'une salle d'apprentissage actif (Céci et Coudrin, 2014; Barrett et al., 2015). Bien que la recherche de Barrett et al. (2015) ait été effectuée auprès 
d'élèves de 5 à 11 ans, il est possible de croire que l'effet de la lumière sur l'apprentissage soit également positif pour les populations adultes (Shishegar et Boubekri, 2016). En plus de cette recension des écrits, une journée de conférences et de discussions avec des experts a permis de poser les assises théoriques du projet.

Cette journée a amené l'équipe d'étudiants à s'appuyer sur les processus cognitifs à la base de l'apprentissage pour réfléchir à la configuration de l'espace. La taxonomie de Bloom révisée par Anderson et Krathwohl (2001) propose une hiérarchisation de ces processus qui sont mémoriser, comprendre, appliquer, analyser, évaluer et créer. L'apprentissage actif permet généralement de viser des objectifs d'apprentissage plus complexes et de mobiliser les processus analyser, évaluer et créer (McDaniel, 2014). À partir des différents processus cognitifs, l'équipe d'étudiants a illustré un maximum de configurations spatiales selon les différentes activités d'apprentissage qui peuvent se dérouler dans l'espace (en s'appuyant sur la typologie de Chamberland et al., 1995). La Figure 2 illustre une partie du travail effectué. Par la disposition du mobilier et la position de l'enseignant (en rouge), il est possible de constater que les niveaux supérieurs de la taxonomie d'Anderson et Krathwohl (2001) peuvent être associés à une interaction accrue entre les apprenants. II est également possible de constater que les apprenants exercent davantage de contrôle lors des activités, un aspect souligné par Normand (2017) notamment.

\section{Figure 2}

Exemples de configurations spatiales en fonction des processus cognitifs et des activités d'apprentissage

Processus cognitifs (Anderson et Krathwohl, 2001)

Mémoriser

Récupérer l'information

Comprendre

Traiter l'information

Appliquer

Exécuter une procédure

Analyser

Identifier les parties d'un tout et comprendre leurs relations
Exemples d'activités d'apprentissage
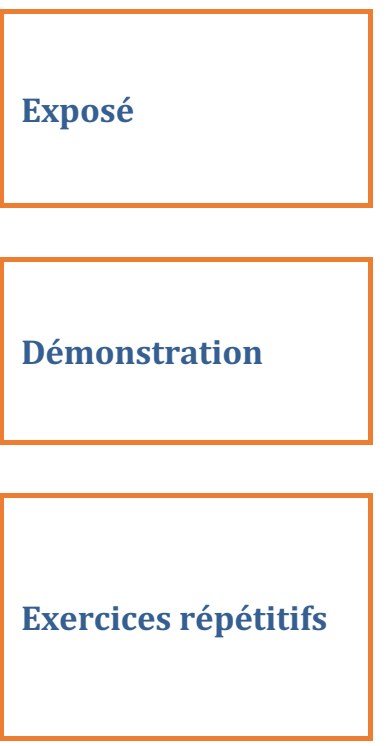

Recherche guidée Étude de cas
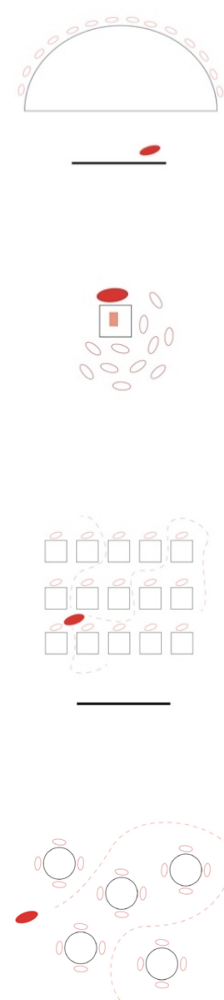
Évaluer

Porter un jugement basé sur des

critères

Créer

Combiner des éléments pour

former un tout cohérent et

original
Groupe de

discussion
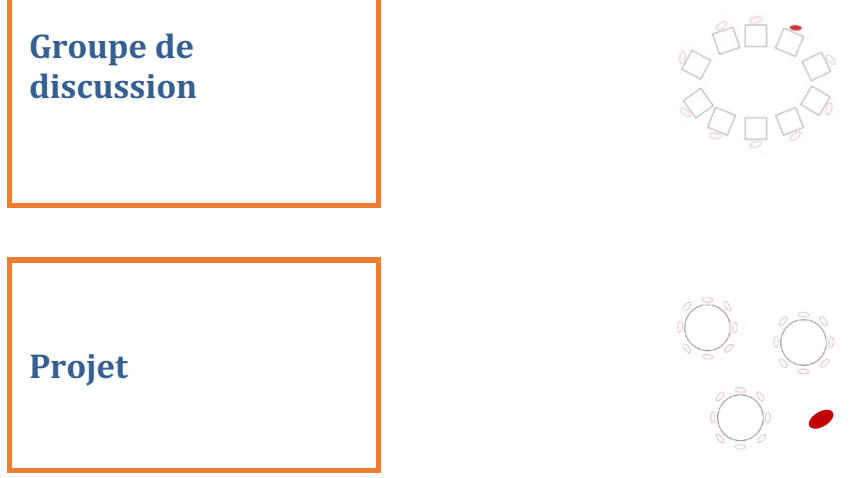

Une fois les différentes activités d'apprentissage et configurations spatiales identifiées, une analyse des contraintes structurelles de l'espace situé au laboratoire informatique de la Faculté des sciences de l'éducation à l'UQAM a été effectuée. De cette analyse a émergé une réflexion quant aux différents éléments de design à considérer dans l'aménagement (zonage de l'espace, rapport à l'extérieur, luminosité, mobilier, accès, etc.). Ces éléments ont été schématisés en tenant compte de l'espace existant. Par la suite, une première esquisse a été produite, puis présentée à l'ensemble des acteurs impliqués dans le projet (professeurs, responsables du service des immeubles, responsables des services informatiques, responsables du service de l'audiovisuel). À partir des rétroactions obtenues, des modifications ont été apportées à l'esquisse, puis trois éléments de l'aménagement à l'intérieur de l'espace ont été approfondis. L'esquisse et ces trois éléments sont présentés dans la section suivante.

\section{Esquisse d'aménagement}

Avant de proposer un aménagement à l'intérieur de la classe, il apparaissait opportun de présenter une vue d'ensemble de la salle dans sa forme actuelle (Figure 3), puis dans sa forme proposée au terme des premières étapes du projet. Une première proposition fusionne les trois locaux en un espace en longueur (Figure 4). Des parois vitrées pouvant s'opacifier au besoin permettent l'accès à la lumière naturelle extérieure, mais aussi une ouverture sur le reste du bâtiment par des parois donnant sur les couloirs. Une deuxième proposition permet un accès direct à la lumière naturelle, mais demanderait une reconfiguration du laboratoire informatique de l'UQAM (Figure 5). 


\section{Figure 3}

Vue d'ensemble des locaux à la disposition du projet (en rose)

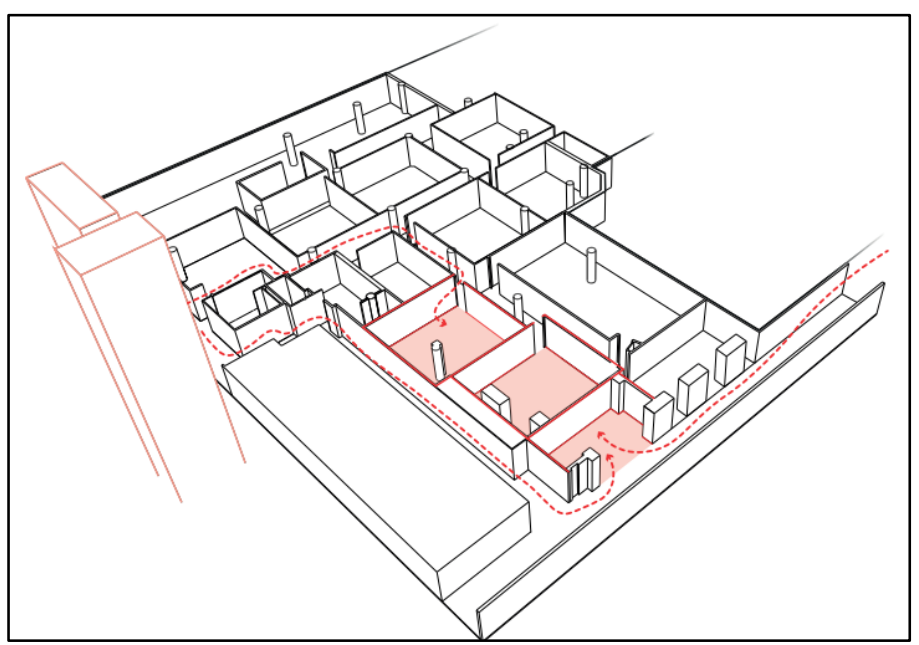

Dans les deux propositions d'aménagement, l'accès aux locaux a été repensé pour conserver l'entrée par un laboratoire informatique de l'UQAM et pour permettre l'entrée par une zone de détente (en gris) où les étudiants pourraient se rassembler. À partir de cette entrée, un vestibule serait aménagé pour que les étudiants y laissent leurs effets personnels.

\section{Figure 4}

Première proposition d'aménagement

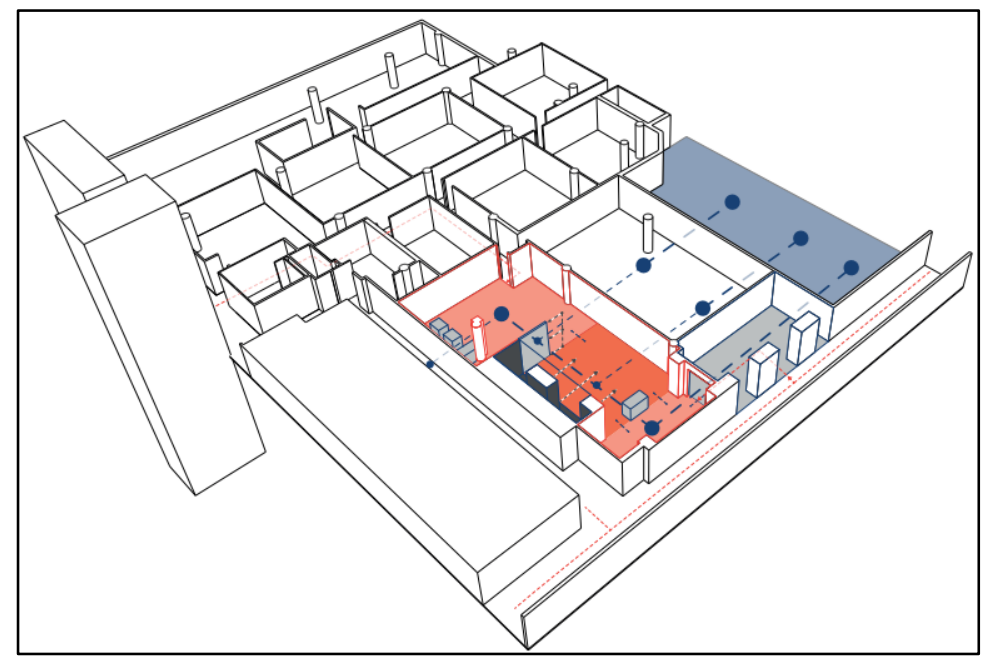




\section{Figure 5}

Deuxième proposition d'aménagement

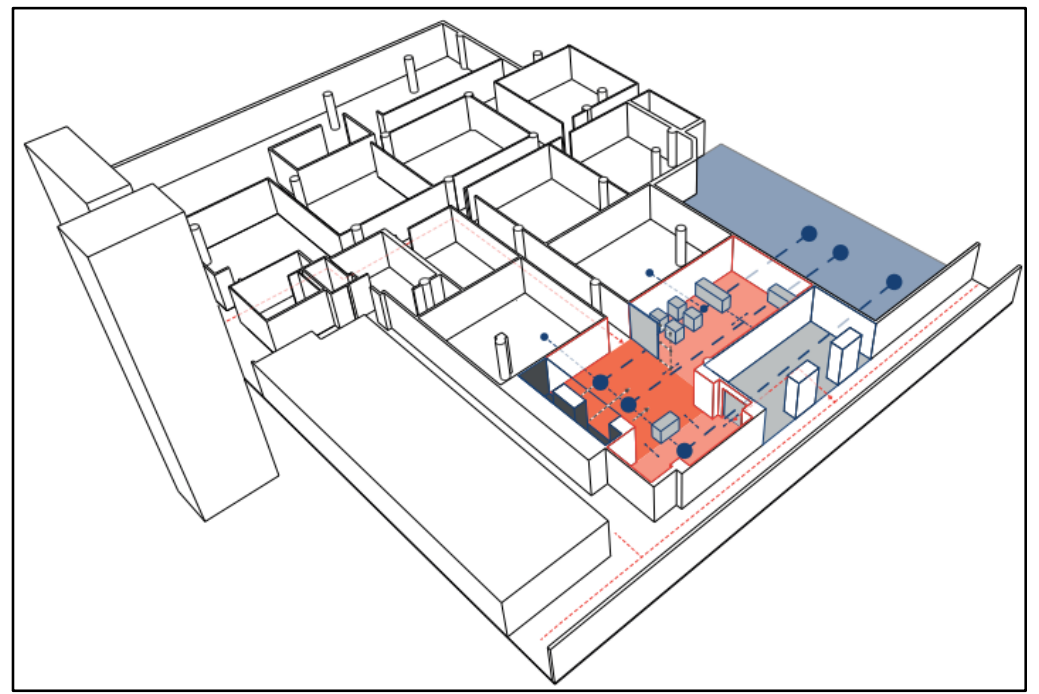

Les différentes observations relevées à l'échelle du bâtiment et à l'échelle des locaux existants ont été associées à la recension des écrits et aux assises théoriques du projet. Trois éléments de l'aménagement à l'intérieur de la classe ont été considérés en particulier : le zonage de l'espace, le cœur technique et le mobilier fixe et mobile. Ces trois éléments sont décrits et illustrés dans les paragraphes suivants.

Le zonage de l'espace est rendu possible par des cloisons mobiles et une perméabilité des parois extérieures au local. Cette division flexible de l'espace permet de mettre en place plusieurs activités d'apprentissage de façon simultanée ou non (Figure 6). Lorsque l'espace reste ouvert, une seule zone favorise le déploiement d'une activité d'apprentissage qui nécessite un grand espace (p. ex. jeu de rôle). Une cloison mobile permet au contraire de segmenter l'espace en plus petites zones de travail (p. ex. travail collaboratif). Comme mentionné précédemment, le vestibule (adjacent à la zone grise) représente un espace plus informel qui peut permettre aux enseignants et aux étudiants de se préparer à faire leur entrée dans la salle. 


\section{Figure 6}

Zonage de l'espace

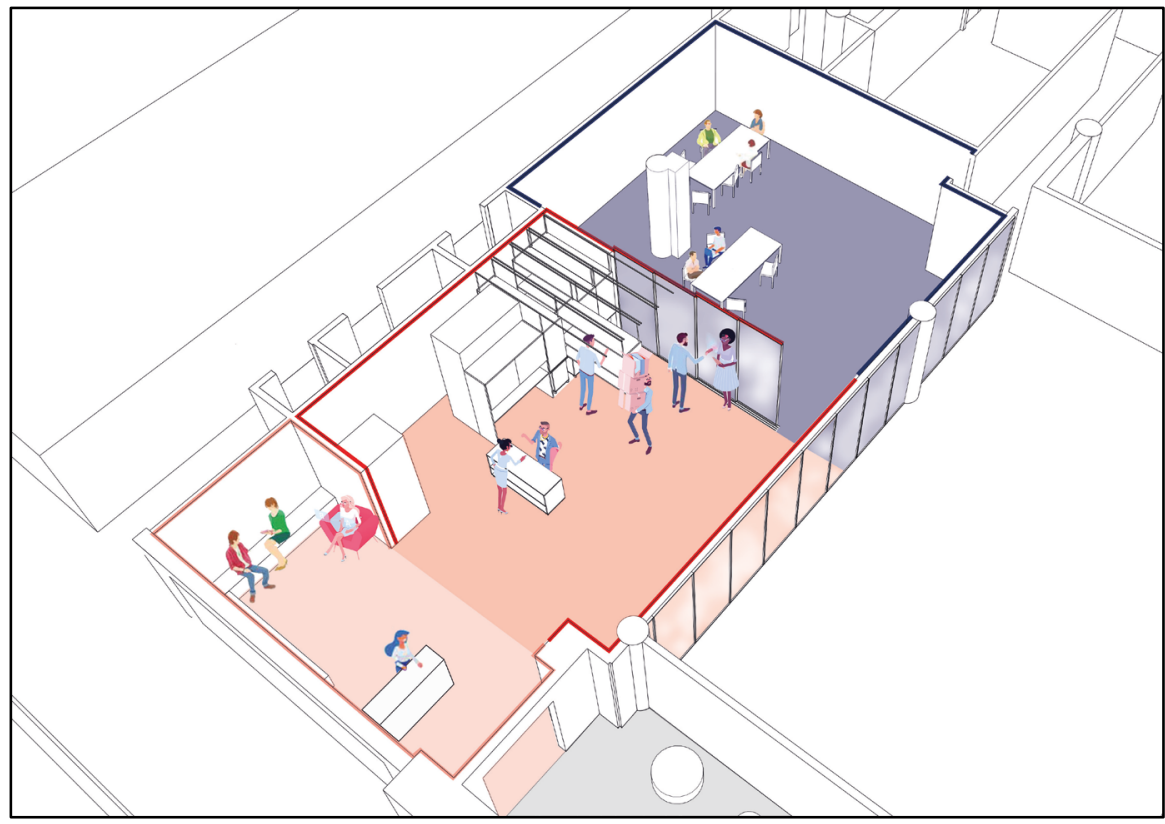

La pertinence d'ajouter une technologie permettant un éclairage flexible (p. ex. un projecteur $360^{\circ}$ ) a également émergé à plusieurs reprises lors du processus de création et de concertation (Figure 7). En effet, en plus de servir de projecteur pour présenter des notions, cet éclairage pourrait également délimiter différentes zones dans la classe.

\section{Figure 7}

Délimitation de l'espace grâce à l'équipement technologique

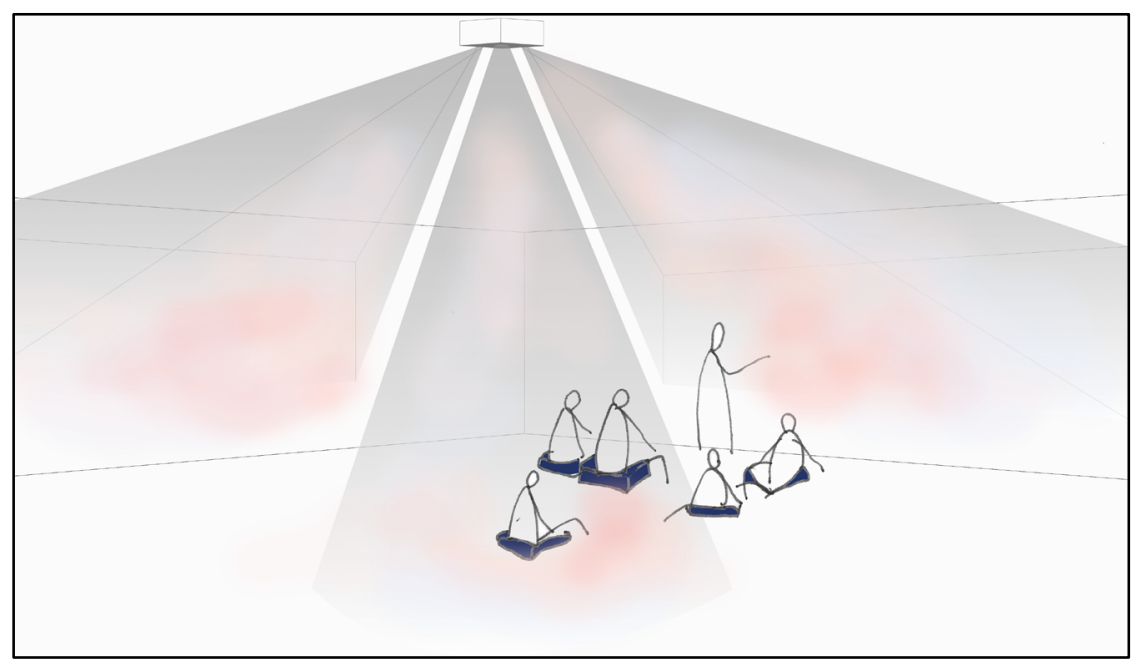


Le deuxième élément est le cœur technique qui est un espace de rangement modulaire (Figure 8). Pour permettre une manipulation adéquate des outils et du mobilier, le cœur technique assure le déploiement du matériel nécessaire aux différentes activités d'apprentissage. Ses dimensions relativement généreuses permettent d'inclure les colonnes structurelles. Divisé en trois parties, il peut être utilisé de plusieurs façons. Une première partie est laissée libre pour ranger du mobilier roulant. Cette partie pourrait évoluer en fonction des besoins changeants. Une deuxième partie correspond aux rangements identifiés en bleu. Ils pourraient accueillir l'équipement technologique (p. ex. imprimante 3D). Une troisième partie identifiée en rouge correspond à des rangements sur roue qui pourraient contenir du mobilier pliable (tables et chaises).

\section{Figure 8}

Cœur technique rangé ( $A$ ) et déployé (B)
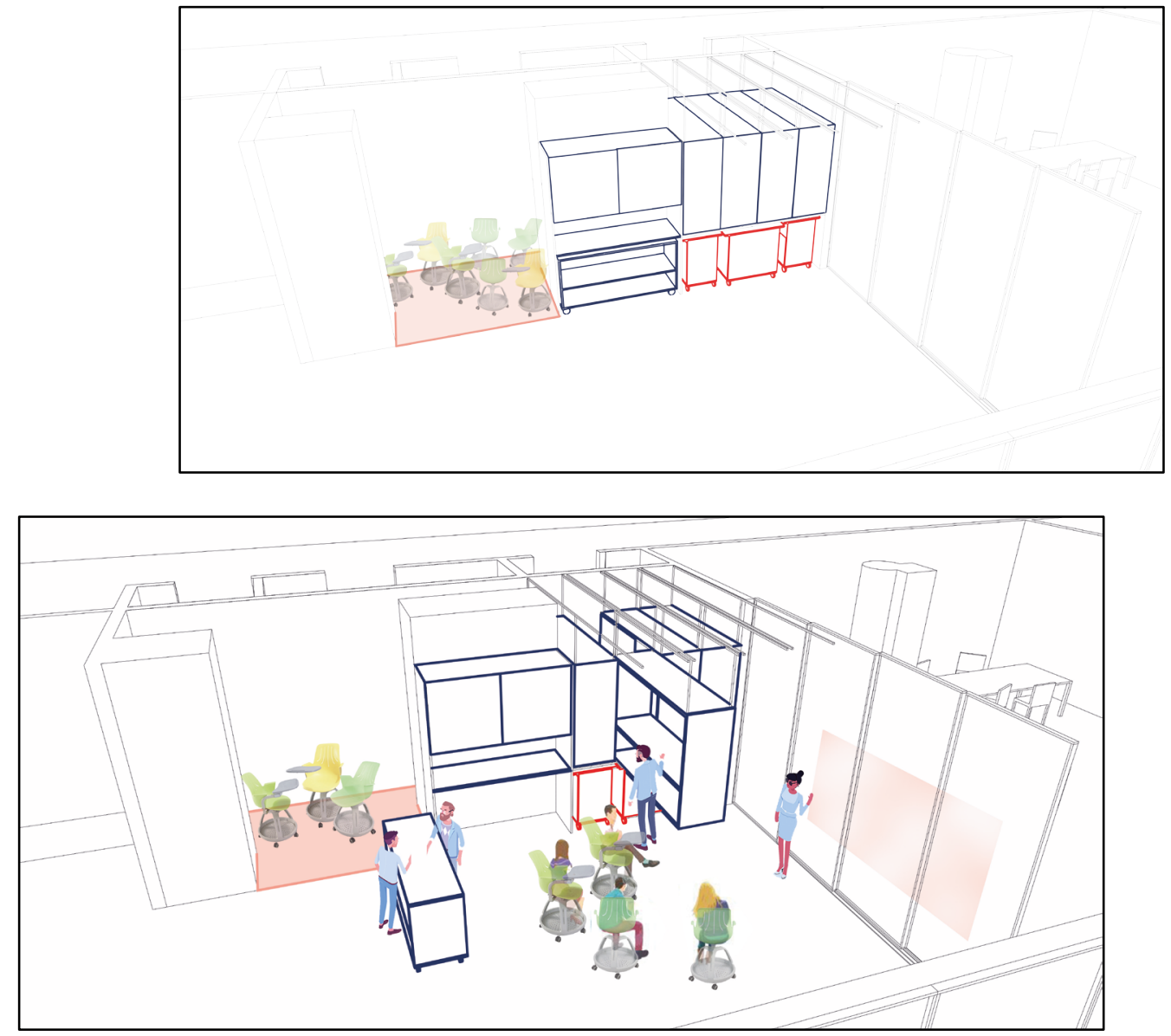

Note. $\mathrm{A}=$ Figure du haut $; \mathrm{B}=$ Figure du bas

Le troisième élément est le mobilier fixe et mobile. Un mobilier fixe a été pensé pour pouvoir utiliser l'espace sans aménagement préalable. Le mobilier mobile se veut, quant à lui, le plus léger et facile possible à installer et à ranger. II permet de mettre en place une variété d'activités d'apprentissage et de passer facilement d'une disposition à l'autre au besoin (p. ex. passer d'une démonstration à un projet en équipe). Le mobilier qui ne peut pas se ranger dans le cœur technique est identifié en gris sur la Figure 9 
et le mobilier qui peut y être rangé est identifié en rouge. Toutes les chaises et les tables sont sur des roues pour permettre une reconfiguration rapide de l'espace.

\section{Figure 9}

Mobilier fixe et mobile

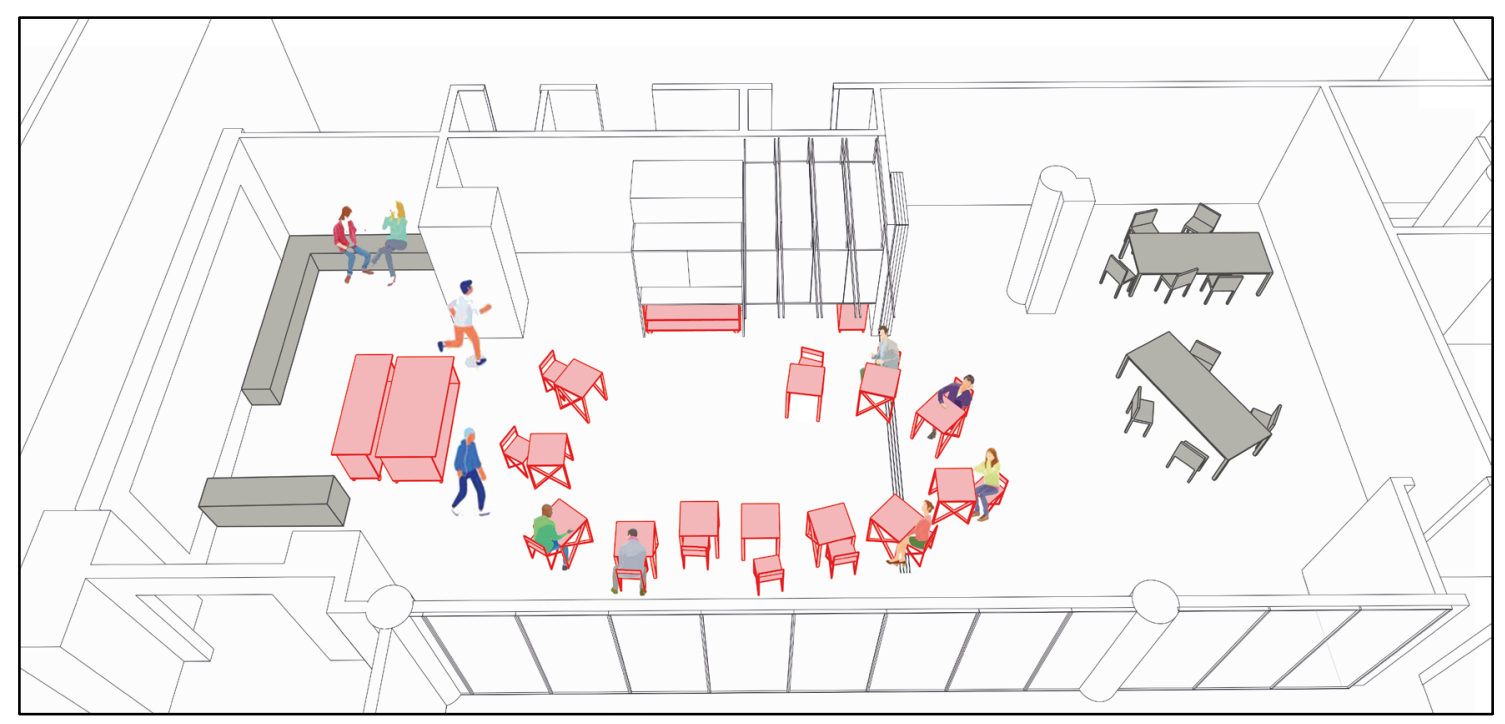

\section{Réflexions et retombées envisageables}

\section{Réflexions sur le processus et sur l'esquisse}

Tant par son processus que par le résultat qui en émerge, le projet de la Classe du futur propose des avenues intéressantes pour la recherche et l'aménagement de classes en milieu universitaire. Le processus de cocréation interdisciplinaire qui s'est déployé dans le cadre du projet se rapproche d'une démarche de recherche-création. Ce type de recherche est utilisé par les disciplines créatives pour définir une activité de recherche académique dont l'aboutissement (la production de connaissances) s'accompagne d'une production artistique (Paquin, 2014). Sans avoir la même envergure, le cas du projet de la Classe du futur s'inscrit tout de même dans cet esprit au niveau du processus et du produit. En effet, un travail de recherche a été fait en amont afin d'orienter la création d'une esquisse d'aménagement. Si la recherche-création est d'abord associée aux disciplines artistiques (Delacourt, 2019), le projet de la Classe $d u$ futur permet de constater que ce type de recherche pourrait s'avérer porteur pour d'autres disciplines. Comme l'aménagement d'une classe mobilise des connaissances en design et en éducation, la collaboration interdisciplinaire apparait tout à fait appropriée pour ce genre de projet. Elle a permis d'adopter un point de vue global de l'aménagement en prenant compte autant des éléments de design que des aspects pédagogiques.

L'apport en lumière naturelle et les accès aux locaux ont été réfléchis dans les deux propositions d'aménagement (voir Figures 4 et 5). Si ces éléments sont énoncés dans les écrits, ils sont plus rarement pris en compte dans les projets d'aménagement. Pourtant, des auteurs soulignent que la lumière naturelle, par son large spectre lumineux et son apport en vitamine $D$, influence positivement le bienêtre et l'apprentissage (Barrett et al., 2015; Shishegar et Boubekri, 2016). De plus, l'esquisse d'aménagement propose un accès par le laboratoire informatique, mais également pas des espaces plus conviviaux. 
Les endroits esthétiques et accueillants auraient un effet bénéfique sur les dimensions motivationnelles et affectives de l'apprentissage (Fournier St-Laurent et al., 2018). Les limites poreuses entre les zones formelles et informelles contribuent à l'expérience d'apprentissage, mais aussi à l'expérience étudiante dans son ensemble (Céci et Coudrin, 2014; Université Laval, 2013). Ces caractéristiques de l'environnement viennent ainsi soutenir l'activité qui se déroule à l'intérieur de la classe. À cet égard, les éléments d'aménagement pris en compte (zonage, cœur technique et mobilier) laissent place à plusieurs possibilités pour diversifier les méthodes pédagogiques ainsi que les modalités d'enseignement. Bien que l'espace proposé laisse présager plusieurs possibilités, ses effets ne pourront être évalués qu'après sa mise en forme. II apparait tout de même opportun de discuter des retombées envisageables de l'aménagement proposé pour la pratique des enseignants.

\section{Retombées envisageables du projet}

Le projet de la Classe du futur cherche à créer des espaces favorisant l'apprentissage actif et l'usage des technologies numériques chez les enseignants en formation. D'abord, la création de sous-espaces grâce aux cloisons et au mobilier pourrait favoriser le travail en sous-groupe et l'interaction (voir Figure 6). Cet aspect est intimement lié à l'apprentissage actif et au développement des compétences du $21^{\mathrm{e}}$ siècle (Joynes et al., 2019). Ensuite, l'accès à des outils variés a été identifié comme des facteurs déterminants dans le développement des compétences du $21^{\mathrm{e}}$ siècle (Lewin et McNicol, 2015) et dans la formation du personnel enseignant (Tondeur et al., 2018). Les espaces de stockage deviennent alors une nécessité (Barrett et al., 2015). C'est pourquoi le concept du cœur technique a été développé (voir Figure 8). Son contenu a cependant été peu réfléchi dans le cadre du projet. Le mobilier mobile et flexible illustré dans l'esquisse (voir Figure 9) est également un aspect souvent évoqué dans les écrits (Céci et Coudrin, 2014; LearningLab Network, 2019). Le rangement d'une partie du mobilier offre une plus grande liberté d'aménagement pour l'enseignant comparativement à d'autres projets existants qui proposent des dispositions limitées du mobilier (p. ex. Fournier St-Laurent et al., 2018). Considérant que l'aménagement de l'espace peut venir dissimuler ses possibilités (Cleveland, 2011; Paquelin, 2017), il apparaissait opportun de proposer un espace dont la flexibilité était accrue. Si l'espace proposé semble offrir un maximum de possibilités, il conviendra de préparer les utilisateurs à optimiser le potentiel de la salle pour l'apprentissage. Dans le contexte de la formation des enseignants, cet espace pourrait donc permettre de réfléchir à l'influence de l'aménagement sur l'apprentissage. Cette réflexion pourrait amener les enseignants à saisir les possibilités de leur propre classe. Bien que l'esquisse proposée dans cet article se situe dans un espace bien précis, plusieurs aspects pourraient être transférables dans d'autres contextes. Par exemple, la disposition des chaises et des bureaux pour permettre la collaboration ou la création de sous-espaces sont possibles peu importe l'environnement de la classe.

Par les caractéristiques énoncées précédemment, le projet de la Classe du futur vise à maximiser le temps en présentiel en offrant un espace qui permet d'expérimenter des activités d'apprentissage, des aménagements de l'espace et des outils numériques variés. Le projet s'inscrit ainsi dans une vision large de l'hybridation en ce sens qu'il vise à combiner plusieurs modalités d'enseignement (Osguthorpe et Graham, 2003; Singh, 2003; Valdès, 1995). La mobilité du mobilier et des cloisons peut permettre de passer rapidement d'activités plus transmissives ( $p$. ex. présentation des consignes, démonstration) à des activités en sous-groupe ( $p$. ex. projet). La création de sous-espaces et la diversification des méthodes pédagogiques favorisent également un enseignement différencié et un rythme individualisé, ce qui constitue un aspect non négligeable pour répondre aux besoins d'une diversité d'apprenants (Beaudoin, 2013; Fournier St-Laurent et al., 2018). L'espace proposé offre également des possibilités pour l'enseignement à distance. Le rangement et le déploiement du matériel offert par le cœur technique pourraient faciliter l'intégration d'outils permettant la création de contenu pour la formation à distance (p. ex. écran vert) ou permettant aux étudiants d'assister à distance à un cours en présentiel (p. ex. robot de téléprésence). De plus, comme l'espace peut se diviser, il serait possible de donner un cours en ligne dans une partie de la salle alors qu'une formation en présentiel se déroule dans une autre section de la classe. 


\section{Conclusion}

Le projet de la Classe du futur propose une esquisse d'aménagement d'une classe dédiée à la formation du personnel enseignant. L'esquisse n'est que la première étape d'un processus complexe qui fera intervenir de nombreux acteurs. Une réflexion sur les moyens de favoriser l'utilisation de la classe et d'évaluer ses effets réels devra notamment être effectuée dans le futur. En effet, il conviendra de favoriser l'adoption de la salle par les utilisateurs en offrant des formations et un accompagnement. Par exemple, des dispositions de l'espace en fonction des activités d'apprentissage pourraient être proposées. De la même manière, la position de l'espace dans un laboratoire informatique n'est pas anodine, puisqu'elle pourrait faciliter l'accès à des ressources matérielles et surtout humaines. La prise en compte de ces aspects contribuera à une utilisation optimale de la salle, ce qui est nécessaire pour mesurer ses effets réels. II est notamment envisagé d'observer la relation entre les différentes caractéristiques de la salle (accès, luminosité, flexibilité du mobilier, zonage de l'espace, disponibilités des ressources dans le cœur technique) et certains aspects de la situation d'enseignement/apprentissage (usage du numérique par les enseignants, diversification des méthodes pédagogiques, motivation et attention des apprenants).

\section{Liste de références}

Anderson, L. et Krathwohl, D. A. (2001). Taxonomy for Learning, Teaching and Assessing: A Revision of Bloom's Taxonomy of Educational Objectives. Longman.

Barrett, P., Zhang, Y., Davies, F. et Barrett, L. (2015). Clever Classrooms: Summary Report of the HEAD Project. Université de Salford. https://usir.salford.ac.uk/id/eprint/35221/

Beaudoin, J. (2013). Introduction aux pratiques d'enseignement inclusives. Université d'Ottawa. https://www.uottawa.ca/respect/sites/www.uottawa.ca.respect/files/accessibilite-guide-inclusion-fr-2013-10-30.pdf

Bonwell, C. et Eison, J. A. (1991). Active Learning : Creating Excitement in the Classroom (ASHE-ERIC Higher Education Report No. 1). The George Washington University, School of Education and Human Development. https://files.eric.ed.gov/fulltext/ED336049.pdf

Céci, J. et Coudrin, D. (2014). (Re)penser les espaces physiques d'apprentissage. Fédération Interuniversitaire de l'Enseignement à Distance. https://doi.org/10.13140/RG.2.2.27110.11841

Chamberland, G., Lavoie, L. et Marquis, D. (1995). 20 formules pédagogiques. Presses de l'Université du Québec.

Cleveland, B. J. (2011). Engaging spaces: Innovative learning environements, pedagogies and student engagement in the middle years of school [Thèse de doctorat]. Université de Melbourne. https://pdfs.semanticscholar.org/e602/1e9e1df68311a7374a600c9ec3fb69a9b700.pdf

Collège LaSalle. (2020). Salles de classe actives. https://www.collegelasalle.com/a-propos/installationsspecialisees/salles-de-classe-actives

Dede, C. (2010). Technological supports for acquiring 21st century skills. Dans E. Baker, B. McGraw et P. Peterson (dir.), International Encyclopedia of Education (3 éd., p. 158-166). Elsevier.

Delacourt, S. (2019). L'artiste-chercheur: Un rêve américain au prisme de Donal Judd. Éditions B42.

Ertmer, P. et Ottenbreit-Leftwich, A. (2013). Removing obstacles to the pedagogical changes required by Jonassen's vision of authentic technology-enabled learning. Computers \& Education, 64, 1-8. https://doi.org/10.1016/j.compedu.2012.10.008

Ertmer, P., Ottenbreit-Leftwich, A. et Tondeur, J. (2015). Teachers' beliefs and uses of technology to support 21st-century teaching and learning. Dans H. Fives et G. Michele (dir.), International handbook of research on teacher beliefs (p. 403-418). Routledge. 
Fournier St-Laurent, S., Normand, L., Bernard, S. et Desrosiers, C. (2018). Les conditions d'efficacité des classes d'apprentissage actif. Collège Ahuntsic. https://eduq.info/xmlui/bitstream/handle/11515/35536/fournier-st-laurentet-al-conditions-efficacite-classes-apprentissage-actif-parea-2018.pdf

Freeman, S., Eddy, S. L., McDonough, M., Smith, M. K., Okoroafor, N., Jordt, H. et Wenderoth, M. P. (2014). Active learning increases student performance in science, engineering, and mathematics. Proceedings of the National Academy of Sciences, 111(23), 8410-8415. https://doi.org/10.1073/pnas.1319030111

Global Partnership for Education (2020). 21st-Century Skills: What potential role for the Global Partnership for Education? A Landscape Review. GPE. https://www.globalpartnership.org/content/21st-century-skills-what-potential-roleglobal-partnership-education

Joynes, C., Rossignoli, S. et Amonoo-Kuofi, E. F. (2019). 21st Century Skills: evidence of issues in definition, demand and delivery for development contexts (K4D Helpdesk Report). Institute of Development Studies. https://assets.publishing.service.gov.uk/media/5d71187ce5274a097c07b985/21st_century.pdf

LearningLab Network. (2019). Les cahiers du Learning Lab Network. LearningLab Network. http://www.learninglabnetwork.com/wp-content/uploads/2019/06/Cahier LLN 1.pdf

Lewin, C. et McNicol, S. (2015). Supporting the development of $21^{\text {st }}$ century skills through ICT. In T. Brinda, N. Reynolds, R. Romeike et A. Schwill (dir.), KEYCIT 2014 : Key competencies in informatics and ICT (p. 98-181). University of Potsdam. https://publishup.uni-potsdam.de/opus4-ubp/frontdoor/deliver/index/docld/7032/file/cid07.pdf

McDaniel, S. (2014). Every Space is a Learning Space : Encouraging informal learning and collaboration in higher education environments. BWBR. https://www.bwbr.com/wp-content/uploads/2020/10/Every-Space-ls-A-Learning-Space WP.pdf

McGill. (2020). Active Learning Classrooms (ALCs). https://www.mcgill.ca/tls/spaces/alc

Normand, L. (2017). L’apprentissage actif: une question de risques... calculés. Pédagogie collégiale, 31(1), 5-12. http://aqpc.qc.ca/sites/default/files/revue/normand-vol.31-1.pdf

Osguthorpe, R. T. et Graham, C. R. (2003). Blended learning environments : Definitions and directions. The Quarterly Review of Distance Education, 4(3), 227-233. https://www.learntechlib.org/p/97576/

Paquelin, D. (2017). Flexibilisation : principes et actions. Site du Bureau de soutien à l'enseignement de l'Université Laval. https://www.enseigner.ulaval.ca/sites/default/files/01-flexibilisation mai 2017.pdf

Paquin, L. C. (2014). La recherche. Méthodologie de la recherche création. http://lcpaquin.com/metho rech creat/index.html

Prince, M. (2004). Does Active Learning Work? A Review of the Research. Journal of Engineering Education, 93(3), 1-9. https://doi.org/10.1002/j.2168-9830.2004.tb00809.x

Shishegar, N. et Boubekri, M. (2016, avril). Natural light and productivity: Analyzing the impacts of daylighting on students' and workers' health and alertness. Communication présentée à l'International Conference on "Health, Biological and Life Science", Istanbul, Turquie.

https://www.researchgate.net/publication/303484362 Natural Light and Productivity Analyzing the Impacts of Daylighting_on_Students'_and_Workers'_Health_and_Alertness

Singh, H. (2003). Building effective blended learning programs. Educational Technology, 43(6), 51-54. http://asianvu.com/digital-library/elearning/blended-learning-by Singh.pdf

Tondeur, J., Aesaert, K., Prestridge, S. et Consuegra, E. (2018). A multilevel analysis of what matters in the training of preservice teacher's ICT competencies. Computers \& Education, 122, 32-42. https://doi.org/10.1016/j.compedu.2018.03.002

Université Laval. (2013). Repenser les espaces physiques d'apprentissage : Orientations stratégies et pédagogiques. Université Laval. https://www.enseigner.ulaval.ca/system/files/espaces_physiques-orientations_strategiques_0.pdf

Valdès, D. (1995). Vers de nouvelles formes de formations : Les formations hybrides (Mémoire de DESS, Université de Paris 2). 